\title{
Acanthaceae endémicas del Perú
}

\section{Blanca León ${ }^{1,2}$}

${ }^{1}$ Museo de Historia Natural, Av. Arenales 1256, Aptdo. 14-0434, Lima 14, Perú

2 Plant Resources Center, University of Texas at Austin, Austin TX 78712 EE.UU.

blanca.leon@mail.utexas.edu

\section{Resumen}

La familia Acanthaceae es reconocida en el Perú por presentar 41 géneros y alrededor de 275 especies (Brako \& Zarucchi, 1993; Ulloa Ulloa et al., 2004), básicamente arbustos y hierbas. En este trabajo reconocemos 42 taxones endémicos en 15 géneros. Tres de estos géneros, Cephalacanthus, Orophochilus y Trichosanchezia, son endémicos al Perú. La mayoría de los taxones endémicos ocupan las regiones Bosques Muy Húmedos Montanos y Bosques Húmedos Amazónicos, entre los 100 y 2870 m de altitud. Siete de los endemismos están representados por lo menos en una área natural protegida.

Palabras claves: Acanthaceae, Cephalacanthus, Orophochilus, Trichosanchezia, Perú, endemismo, plantas endémicas.

\section{Abstract}

The Acanthaceae are represented in Peru by 41 genera and approximately 275 species (Brako \& Zarucchi, 1993; Ulloa Ulloa et al., 2004), basically shrubs and herbs. Here we recognize 42 endemic taxa in 15 genera. Three genera, Cephalacanthus, Orophochilus and Trichosanchezia, are endemic to Peru. Most endemic Acanthaceae are found in the Very Humid Montane and Humid Lowland Amazonian Forests regions, between 100 and $2870 \mathrm{~m}$ elevation. Seven endemic species are represented within Peru's protected areas system.

Keywords: Acanthaceae, Cephalacanthus, Orophochilus, Trichosanchezia, Peru, endemism, endemic plants.

\section{Aphelandra cuscoensis Wassh.}

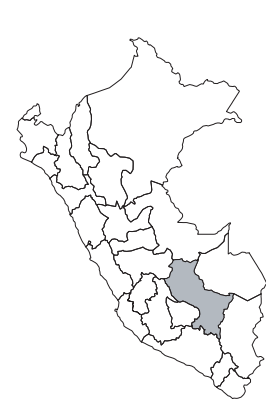

\section{EN, B1a}

Publicación: Phytologia 25(7): 469—470. 1973.

Colector: C. Vargas C. 15415

Herbarios: (US)

Nombre común: Desconocido.

Registro departamental: CU.

Regiones Ecológicas: BHA; 470—520 m. SINANPE: PNM

Herbarios peruanos: CUZ (1).

Observaciones: Hierba terrestre conocida solamente del sur del país, de las cuencas del Marcapata y del Madre de Dios. Aparentemente no ha vuelto a ser recolectada desde la década de 1960.

\section{Aphelandra hapala Wassh.}

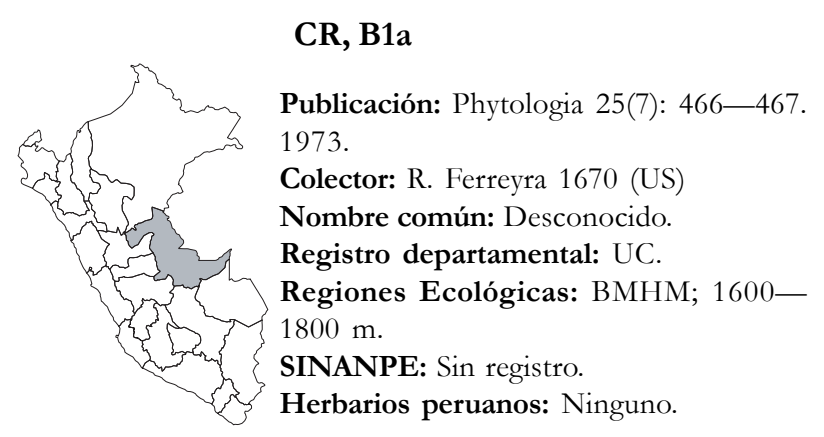

Observaciones: Hierba terrestre conocida aparentemente de la colección tipo, una planta recolectada en 1947 de la cuenca del Aguaytía. Podría estar representada en el Parque Nacional Cordillera Azul.

\section{Aphelandra juninensis Wassh.}

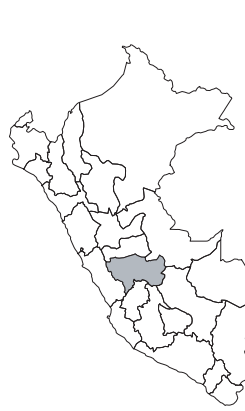

\section{CR, B1ab(iii)}

Publicación: Phytologia 25(7): 471—472. 1973.

Colector: A. Weberbauer 6537 (F; MOL!)

Nombre común: Desconocido.

Registro departamental: JU.

Regiones Ecológicas: MA; 2000—3000 $\mathrm{m}$.

SINANPE: Sin registro.

Herbarios peruanos: MOL (isotipo).

Observaciones: Arbusto conocido solamente de la colección tipo, una planta recolectada en 1913 en la cuenca del Pariahuanca, un tributario del Mantaro. Weberbauer (1945) describe esta zona como un ambiente ecotonal de matorrales y bosques de ceja en quebradas húmedas. Esta zona requiere de exploración y estudio botánico.

\section{Aphelandra latibracteata Wassh.}

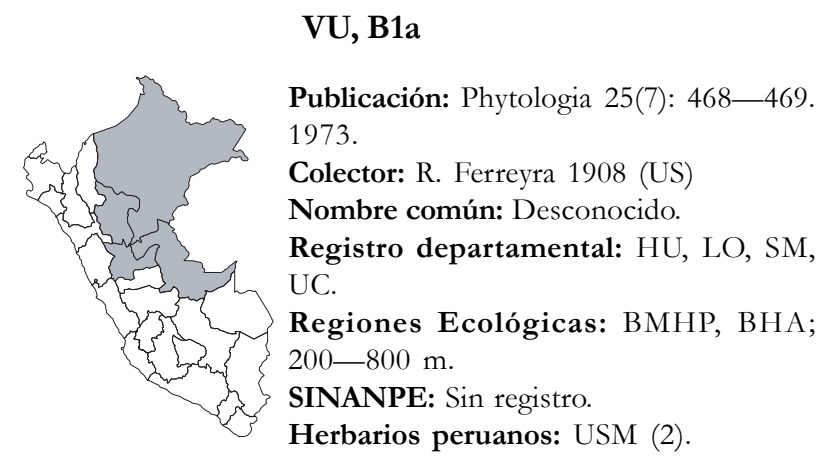

Observaciones: Arbusto recolectado en varias localidades de las cuencas del Pachitea, Ucayali y Amazonas. Una de las localidades recolectadas está deteriorada por la expansión urbana y rural; esta tendencia se observa para las otras localidades. 


\section{Aphelandra viscosa Mildbr.}

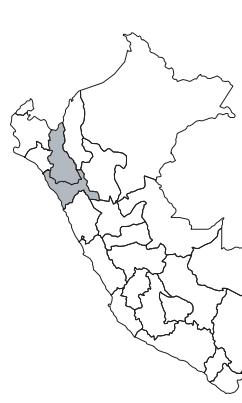

\section{EN, B1a}

Publicación: Repert. Spec. Nov. Regni Veg. 11: 66. 1930.

Colector: A. Raimondi 7133

Herbarios: (B)

Nombre común: Desconocido. Registro departamental: CA, LL. Regiones Ecológicas: MA; 1950—2700 $\mathrm{m}$.

SINANPE: Sin registro.

Herbarios peruanos: HAO (2), HUT (2).

Observaciones: Esta especie leñosa es conocida de poblaciones en las vertientes del Pacífico del norte del país. Sus poblaciones están naturalmente fragmentadas en las cuencas del Chicama y Magdalena.

\section{Aphelandra weberbaueri Mildbr.}

\section{EN, B1a}



Publicación: Notizbl. Bot. Gart. BerlinDahlem 11: 67. 1930.

Colector: A. Weberbauer 6657 (B; MOL!)

Nombre común: Desconocido.

Registro departamental: JU.

Regiones Ecológicas: BMHM, BMHP; 1000-1700 m.

SINANPE: Sin registro.

Herbarios peruanos: MOL (isotipo).

Observaciones: Esta especie arbustiva es conocida solamente del centro del país de las cuencas del Tulumayo y Masamerich, afluentes del Perené. Aparentemente no ha vuelto a ser recolectada de la localidad original, un área escasamente herborizada y afectada por la expansión agrícola.

\section{Cephalacanthus maculatus Lindau}



\section{CR, B1ab(iii)}

Publicación: Repert. Spec. Nov. Regni Veg. 1: 158.1905.

Colector: A. Weberbauer 4647 (B; MOL!)

Nombre común: Desconocido.

Registro departamental: SM.

Regiones Ecológicas: BMHP; $1400 \mathrm{~m}$.

SINANPE: Sin registro.

Herbarios peruanos: MOL (isotipo).

Observaciones: Esta especie es conocida solamente de la colección tipo, una planta recolectada en 1904 en la cuenca del Mayo. Podría estar representada en el Bosque de Protección del Alto Mayo. Esta cuenca está siendo modificada para acomodar la ampliación agrícola.

\section{Dicliptera porphyrea Lindau}

\section{EN, B1ab(iii)}

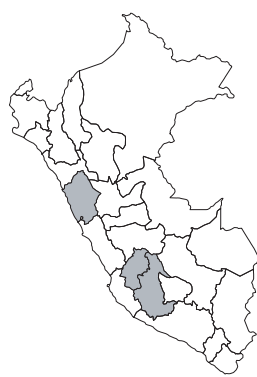

Publicación: Bull. Herb. Boissier, ser. 2, 5(4): 368-369. 1905.

Colector: A. Weberbauer 2676 (B; MOL!)

Nombre común: Desconocido.

Registro departamental: AN, AY, HV.

Regiones Ecológicas: MA; 3200—3400

$\mathrm{m}$.

SINANPE: Sin registro.

Herbarios peruanos: MOL (isotipo).
Observaciones: Esta especie es conocida solamente de cuencas que drenan al Pacífico. Esta especie podría estar afectada por los incendios intencionales.

\section{Dicliptera rauhii Wassh.}

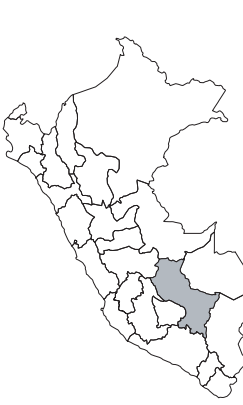

CR, B1ab(iii)

Publicación: Beitr. Biol. Pflanzen 63: 425. 1988.

Colector: W. Rauh \& C. Hirsch P. P804

Herbarios: (HEID)

Nombre común: Desconocido.

Registro departamental: CU.

Regiones Ecológicas: BMHM; $2500 \mathrm{~m}$.

SINANPE: SHMP

Herbarios peruanos: Ninguno.

Observaciones: Esta hierba terrestre es conocida aparentemente sólo de la colección tipo, una planta recolectada en 1954 en la cuenca del Urubamba. Esta especie podría estar afectada por los incendios intencionales fuera del Santuario que se extienden a este.

\section{Justicia manserichensis Wassh.}

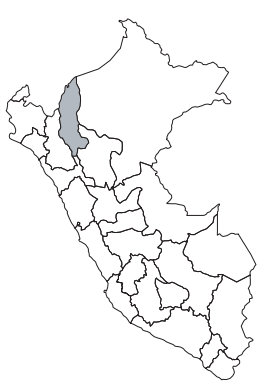

\section{EN, B1ab(iii)}

Publicación: BioLlania, Ed. Especial 6: 545-546, f. 2. 1997.

Colector: J.J. Wurdack 2284 (US; USM!)

Nombre común: Desconocido.

Registro departamental: AM.

Regiones Ecológicas: BHA; 240—450 m. SINANPE: ZRSC

Herbarios peruanos: HUT (4), USM (isotipo+9).

Observaciones: Esta especie herbácea es conocida de las cuencas del Santiago y Cenepa. La colección tipo proviene de la Cordillera de Campanquiz en el límite entre los departamentos de Amazonas y Loreto. Esta cordillera aislada ha sido escasamente herborizada. Amenazas a sus poblaciones provienen de la expansión agrícola.

\section{Justicia elegantissima (Lindau) Wassh.}

\section{EN, B1ab(iii)}

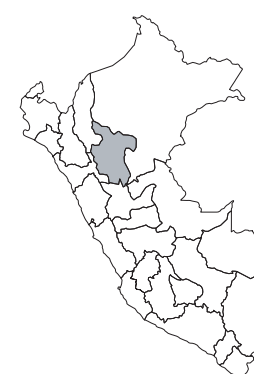

Publicación: Monogr. Syst. Bot. Missouri Bot. Gard. 45: 1253. 1993.

Colector: E.H.G. Ule 6289 (B)

Nombre común: Desconocido.

Registro departamental: SM.

Regiones Ecológicas: BMHM; 900$1100 \mathrm{~m}$.

SINANPE: Sin registro.

Herbarios peruanos: MOL (1).

Observaciones: Arbusto conocido aparentemente sólo de dos localidades en el nor-oriente del país. La localidad tipo proviene de la cuenca del Ponasa, una zona escasamente herborizada y que alberga varios endemismos. Brako \& Zarucchi (1993) citaron Ucayali como parte de la distribución de esta especie, pero no se halló colecciones de herbario provenientes de ahí. Esta especie también es conocida de la cuenca del Mayo. 


\section{Justicia loretensis Lindau}

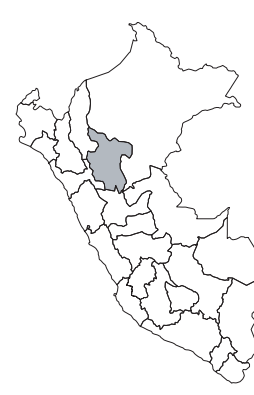

EN, B1ab(iii)

Publicación: Bull. Herb. Boissier, ser. 2, 4: 410. 1904.

Colector: E.H.G. Ule 6876 (B)

Nombre común: Desconocido.

Registro departamental: SM.

Regiones Ecológicas: BMHP; altitud desconocida.

SINANPE: ACRCE

Herbarios peruanos: Ninguno.

Observaciones: Arbusto conocido de dos localidades en el nororiente del país. La colección tipo fue recolectada en 1904 de la cuenca del Mayo. Esta zona alberga numerosos endemismos y la localidad original no ha sido extensamente herborizada. Esta localidad forma hoy parte del Area de Conservación Regional Cerro La Escalera. Esta especie fue citada como proveniente de Loreto, pero no hay registro de ese departamento. Esta especie también es conocida de la cuenca del Huallaga.

\section{Justicia weberbaueri (Lindau) Wassh.}

\section{EN, B1ab(iii)}

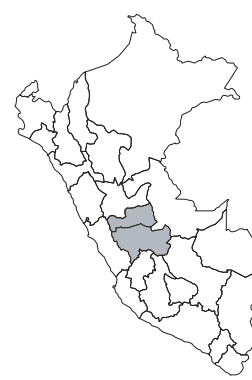

Publicación: Monogr. Syst. Bot. Missouri Bot. Gard. 45: 1253. 1993.

Colector: A. Weberbauer 6557

Herbarios: (B; $\underline{\mathrm{MOL} !})$

Nombre común: Desconocido.

Registro departamental: JU, PA.

Regiones Ecológicas: MA, BS; 2200$2300 \mathrm{~m}$.

SINANPE: Sin registro.

Herbarios peruanos: MOL (isotipo), MOLF (1), USM (1).

Observaciones: Arbusto conocido solamente del centro del país en las cuencas del Mantaro y del Pozuzo. La colección tipo fue recolectada en 1913 de una zona con comunidades xerofíticas (Weberbauer, 1945). Tanto esa parte del Mantaro como las comunidades xéricas requieren de mayor exploración y herborización. Los incendios intencionales podrían afectar esta especie.

\section{Justicia alpina Lindau}

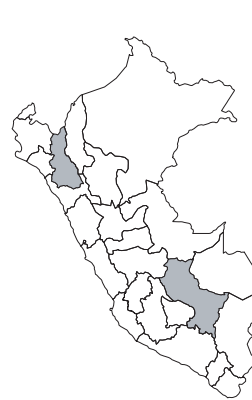

\section{VU, B1a}

Publicación: Repert. Spec. Nov. Regni Veg. 1: 159. 1905

Colector: A. Weberbauer 3817 (B; MOL!)

Nombre común: Desconocido.

Registro departamental: CA, CU.

Regiones Ecológicas: MA, BMHM; 2000-2870 m.

SINANPE: SHMP

Herbarios peruanos: CPUN (2), HAO (2), HUT (2), MOL (isotipo), USM (1).

Observaciones: Hierba terrestre descrita de una planta recolectada en la cuenca del Magdalena en el norte de la vertiente del Pacífico. También se halla en la cuenca del Cajamarca. Esta especie es conocida además de poblaciones disyuntas en el sur de la vertiente oriental.

\section{Mendoncia peruviana Leonard}

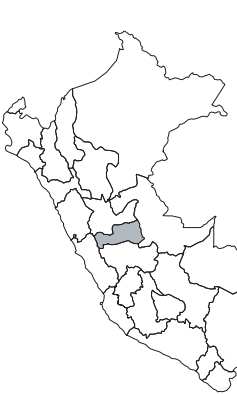

CR, B1ab(iii)

Publicación: J. Wash. Acad. Sci. 21(7): 148. 1931.

Colector: E.P. Killip \& A.C. Smith 26357 (NY, US)

Nombre común: Desconocido.

Registro departamental: PA.

Regiones Ecológicas: BHA; $400 \mathrm{~m}$.

SINANPE: Sin registro.

Herbarios peruanos: Ninguno.

Observaciones: Este bejuco es conocido aparentemente sólo de la colección tipo, una planta recolectada en 1929 de la cuenca del Pichis. Esta especie fue hallada en matorrales ribereños, un tipo de ambiente escasamente herborizado, al mismo tiempo fácilmente impactado por procesos naturales o actividades humanas.

\section{Mendoncia tarapotana Lindau}

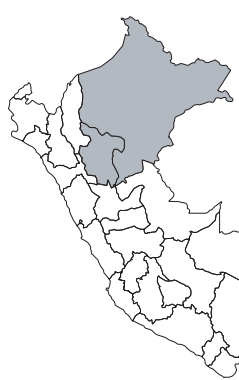

\section{EN, B1a}

Publicación: Bull. Herb. Boissier, ser. 2, 4: 313. 1904.

Colector: R. Spruce 4620 (B, K)

Nombre común: Desconocido.

Registro departamental: LO, SM.

Regiones Ecológicas: BMHP, BHA; $110-700 \mathrm{~m}$.

SINANPE: Sin registro.

Herbarios peruanos: USM (3).

Observaciones: Bejuco conocido de dos localidades aisladas entre ellas por más de $350 \mathrm{~km}$ en línea recta. La localidad tipo está ubicada en la cuenca del Mayo, mientras que la otra está en la del Itaya, en ambientes pantanosos. Aparentemente no ha vuelto a ser recolectada desde 1974

\section{Odontophyllum cuscoensis (Wassh.) Sreem.}

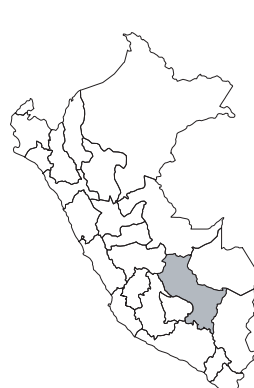

\section{CR, B1a}

Publicación: Phytologia 37(4): 414. 1977. Colector: C. Vargas C. 15415

Herbarios: (US)

Nombre común: Desconocido.

Registro departamental: CU.

Regiones Ecológicas: BHA; $520 \mathrm{~m}$.

SINANPE: Sin registro.

Herbarios peruanos: Ninguno.

Observaciones: Arbusto recolectado en 1964 de la cuenca del Marcapata. Aparentemente no hay recolectas adicionales.

\section{Oplonia hutchisonii Wassh.}

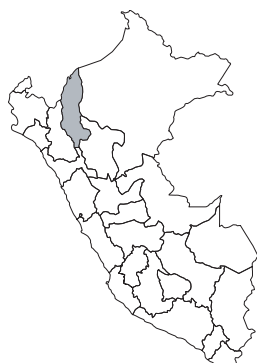

\section{CR, B1a}

Publicación: Phytologia 33: 444. 1976. Colector: P.C. Hutchison 1559a (US)

Nombre común: Desconocido.

Registro departamental: AM.

Regiones Ecológicas: BHA; $400 \mathrm{~m}$.

SINANPE: Sin registro.

Herbarios peruanos: Ninguno.

Observaciones: Arbusto conocido solamente de la localidad tipo entre las cuencas del Marañón y del Imaza. Esta especie fue recolectada en 1958. 


\section{Orophochilus stipulaceus Lindau}

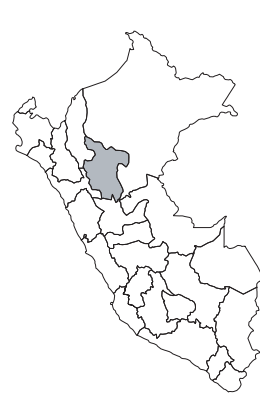

\section{CR, B1ab(iii)} 1897. desconocida.
Publicación: Bull. Herb. Boissier 5(8): 658.

Colector: R. Spruce 4324 (B, NY)

Nombre común: Desconocido.

Registro departamental: SM.

Regiones Ecológicas: BMHP; altitud

SINANPE: Sin registro.

Herbarios peruanos: Ninguno.

Observaciones: Arbusto conocido solamente de la colección tipo, una planta recolectada en el siglo XIX de la cuenca del Mayo. Aparentemente no ha vuelto a ser recolectada. Esta parte del país está siendo transformada por la expansión agrícola.

\section{Pachystachys killipii Wassh.}

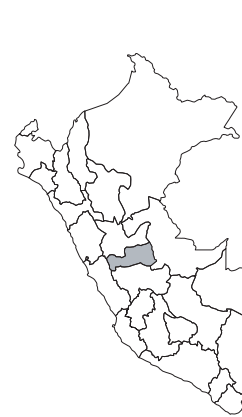

\section{CR, B1ab(iii)}

Publicación: Proc. Biol. Soc. Wash. 99: 179. 1986.

Colector: E.P. Killip \& A.C. Smith 26621 (NY, US)

Nombre común: Desconocido.

Registro departamental: PA.

Regiones Ecológicas: BHA; $375 \mathrm{~m}$.

SINANPE: Sin registro.

Herbarios peruanos: Ninguno.

Observaciones: Arbusto conocido aparentemente sólo de la colección tipo proveniente de la cuenca del Pichis. Brako \& Zarucchi (1993) listaron esta especie para Junín, pero en realidad la localidad está en el Departamento de Pasco. Esta especie fue recolectada en 1929, cuando el acceso a esta parte de la cuenca del Pichis era por río o trocha peatonal. Podría estar representada en el Parque Nacional Yanachaga-Chemillén.

\section{Pachystachys incarnata Wassh.}

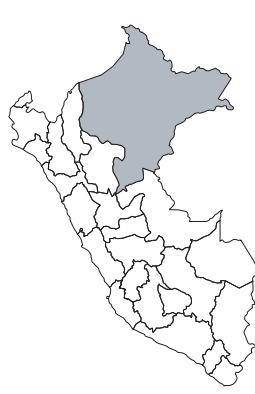

\section{CR, B1ab(iii)}

Publicación: Proc. Biol. Soc. Wash. 99: 171. 1986.

Colector: E.P. Killip \& A.C. Smith 28926

Herbarios: (US)

Nombre común: Desconocido.

Registro departamental: LO.

Regiones Ecológicas: BHA; $135 \mathrm{~m}$.

SINANPE: Sin registro.

Herbarios peruanos: Ninguno.

Observaciones: Este especie arbustiva es conocida aparentemente sólo de la colección tipo, una planta recolectada en 1929, cerca de Yurimaguas, en la cuenca del Huallaga. Los ambientes naturales de esta parte del país están afectados por la expansión agrícola.

\section{Pachystachys longibracteata Wassh.}

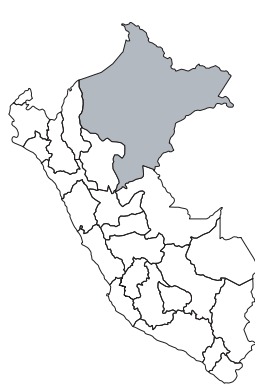

\section{CR, B1ab(iii)}

Publicación: Proc. Biol. Soc. Wash. 99: 163. 1986.

Colector: E.P. Killip \& A.C. Smith 28725 (US)

Nombre común: Desconocido.

Registro departamental: LO.

Regiones Ecológicas: BHA; $135 \mathrm{~m}$.

SINANPE: Sin registro.

Herbarios peruanos: Ninguno.
Observaciones: Especie arbustiva aparentemente conocida sólo de la colección tipo. Esta especie fue recolectada en 1929 de la cuenca del Huallaga. Esta zona alberga varios endemismos. La expansión agrícola es el factor de transformación de los bosques en esta cuenca.

\section{Pachystachys puberula Wassh.}

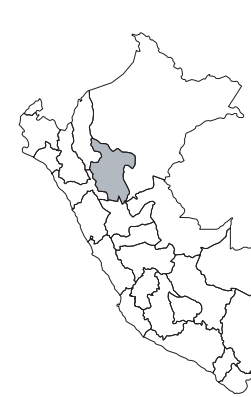

\section{EN, B1a}

Publicación: Proc. Biol. Soc. Wash. 99: 173. 1986.

Colector: D. Wasshausen \& F. Encarnación 1023 (US)

Nombre común: Desconocido.

Registro departamental: SM.

Regiones Ecológicas: BHA; $200-800 \mathrm{~m}$. SINANPE: Sin registro.

Herbarios peruanos: USM (13).

Observaciones: Arbusto conocido de la cuenca del Huallaga. Ha sido recolectada en varias oportunidades de matorrales y márgenes de bosques intervenidos. Esta especie fue listada por Bridgewater et al. (2003) como parte de la flora de los bosques estacionalmente secos.

\section{Pachystachys schunkei Wassh.}

\section{CR, B1ab(iii)}

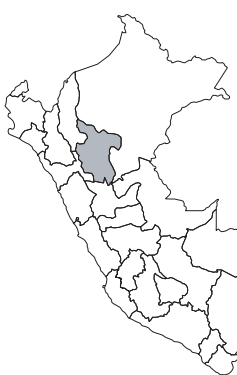

Publicación: Proc. Biol. Soc. Wash. 99: 166. 1986.

Colector: J. Schunke V. 7597 (US)

Nombre común: Desconocido.

Registro departamental: SM.

Regiones Ecológicas: BMHP, BHA; 500-700 m.

SINANPE: Sin registro.

Herbarios peruanos: Ninguno.

Observaciones: Arbusto conocido aparentemente sólo de la colección tipo, una planta proveniente de la cuenca del Huallaga. Esta parte de la cuenca está afectada por la actividad agrícola.

\section{Pachystachys fosteri Wassh.}

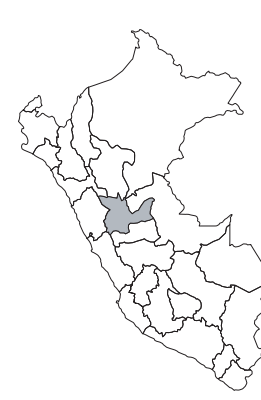

\section{CR, B1ab(iii)}

Publicación: Proc. Biol. Soc. Wash. 99: 168. 1986

Colector: R.B. Foster 8686

Herbarios: (F, US; USM!)

Nombre común: Desconocido.

Registro departamental: HU.

Regiones Ecológicas: BHA; 250—300 m.

SINANPE: Sin registro.

Herbarios peruanos: USM (isotipo).

Observaciones: Arbusto conocido aparentemente sólo de la colección tipo, una planta recolectada en 1982 en la cuenca del Pachitea. Desde entonces esta parte de la cuenca está siendo transformada por las actividades agrícolas y mineras. 


\section{Ruellia rauhii Wassh.}

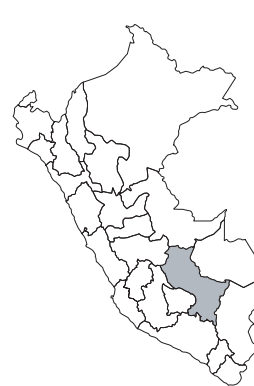

\section{CR, B1a}

Publicación: Beitr. Biol. Pflanzen 63: 421-423. 1988.

Colector: D. Wasshausen \& F. Encarnación 581 (G, K, MO, NY, US)

Nombre común: Desconocido.

Registro departamental: CU.

Regiones Ecológicas: BMHP, BHA; $600 \mathrm{~m}$.

SINANPE: Sin registro.

Herbarios peruanos: Ninguno.

Observaciones: Hierba perenne conocida aparentemente sólo de una localidad en la cuenca del Cosñipata. Por la información del ejemplar tipo se conoce que habita ambientes ribereños. Estos ambientes reciben escasa herborización.

\section{Sanchezia tarapotensis Leonard \& L.B. Sm.}



\section{EN, B1a}

Publicación: Rhodora 66(768): 341, f. 5JL. 1964.

Colector: R. Ferreyra 7883 (US; USM!)

Nombre común: Desconocido.

Registro departamental: AM, SM.

Regiones Ecológicas: BHA; 260—500 m.

SINANPE: Sin registro.

Herbarios peruanos: HUT (2), USM (isotipo).

Observaciones: Esta especie herbácea fue descrita de una planta recolectada en la cuenca del Mayo en 1950. También se conoce de la cuenca del Imaza, en donde ha sido recolectada recientemente (1997).

\section{Sanchezia villosa Leonard \& L.B. Sm.}

\section{CR, B1ab(iii)}

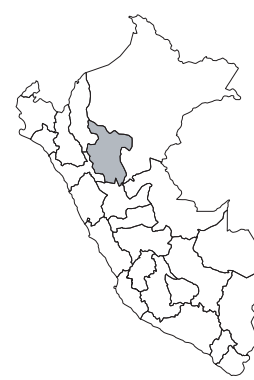

Publicación: Rhodora 66(768): 328-329, f. 3A. 1964.

Colector: R. Ferreyra 7969 (US; MOL! USM!)

Nombre común: Desconocido,

Registro departamental: SM.

Regiones Ecológicas: BMHP; $600-800$ $\mathrm{m}$.

SINANPE: Sin registro.

Herbarios peruanos: MOL (isotipo), USM (isotipo).

Observaciones: Planta perenne conocida aparentemente sólo de la colección tipo, una planta recolectada en 1950 entre la cuenca del Mayo y del Huallaga. No se conoce detalles de su hábitat, pero en esa parte de San Martín los ambientes boscosos están siendo deforestados para la ampliación de la frontera agrícola.

\section{Sanchezia wurdackii Wassh.}

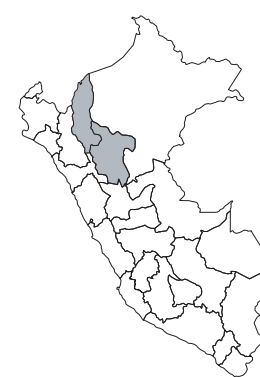

EN, B1a

Publicación: BioLlania, Ed. Especial 6: 543. 1997.

Colector: J.J. Wurdack 2051

Herbarios: (US; USM!)

Nombre común: Desconocido.

Registro departamental: AM, SM.

Regiones Ecológicas: BHA; $320 — 500 \mathrm{~m}$.

SINANPE: Sin registro.

Herbarios peruanos: USM (isotipo+1).
Observaciones: Esta hierba perenne es conocida de la cuenca de las cuencas del Imaza, Mayo y Marañón. El ejemplar tipo fue recolectado en 1962 y, aparentemente, no ha vuelto a ser registrado en esa localidad.

\section{Sanchezia sanmartinensis Leonard \& L.B. Sm.}

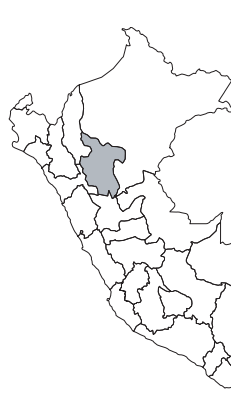

$$
\text { EN, B1ab(iii) }
$$

Publicación: Rhodora 66(768): 328, f. 2HI. 1964.

Colector: L. Williams 6018 (F, US)

Nombre común: Desconocido.

Registro departamental: SM.

Regiones Ecológicas: BMHP, BHA; $300-750 \mathrm{~m}$

SINANPE: Sin registro.

Herbarios peruanos: USM (1).

Observaciones: Arbusto conocido de aparentemente de tres localidades en las cuencas del Mayo y del Huallaga. La colección tipo fue recolectado en 1929 de los alrededores de Tarapoto. Probablemente las actividades agrícolas sean un problema para esta especie.

\section{Sanchezia filamentosa Lindau}

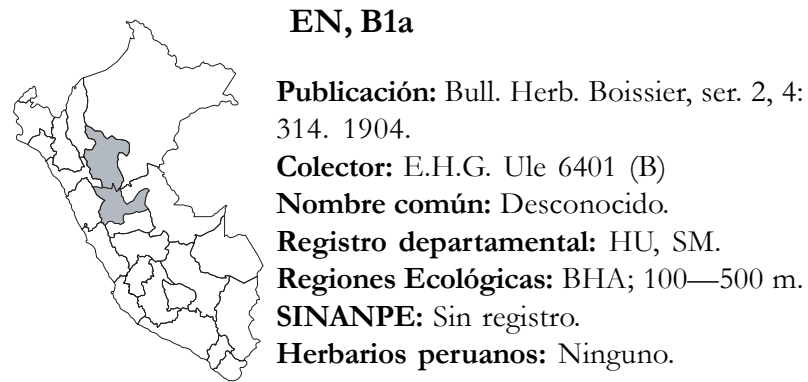

Observaciones: Arbusto conocido de aparentemente dos localidades en el nor-oriente del país. La colección tipo fue recolectada en 1902 en la cuenca del Cainarachi, afluente del Huallaga, en el departamento de San Martín y no Loreto. como fue registrada por Brako \& Zarucchi (1993).

\section{Sanchezia capitata (Nees) Lindau}

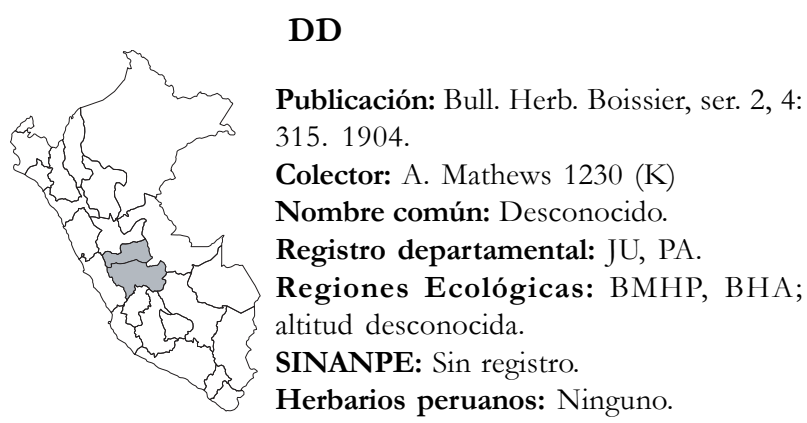

Observaciones: Esta especie arbustiva es conocida de dos localidades. La colección tipo fue recolectada en la cuenca del Pangoa en el siglo XVIII. Probablemente la expansión urbana y las actividades agrícolas sean problemas para esta especie. 


\section{Sanchezia bicolor Leonard \& L.B. Sm.}

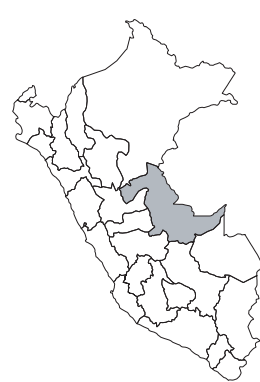

$$
\text { CR, B1ab(iii) }
$$

Publicación: Rhodora 66(768): 320—321, f. 1E-F. 1964.

Colector: H.A. Allard 21711

Herbarios: (US)

Nombre común: Desconocido.

Registro departamental: UC.

Regiones Ecológicas: BHA; $410 \mathrm{~m}$.

SINANPE: Sin registro.

Herbarios peruanos: Ninguno.

Observaciones: Esta especie herbácea es conocida solamente de la colección tipo, una planta recolectada en el límite entre los departamentos de Huánuco y Ucayali, en la cuenca del Aguaytía. Esta zona alberga varios endemismos. Brako \& Zarucchi (1993) citaron San Martín, pero esto es un error. Podría estar representada en el Parque Nacional Cordillera Azul.

\section{Sanchezia williamsii Leonard}

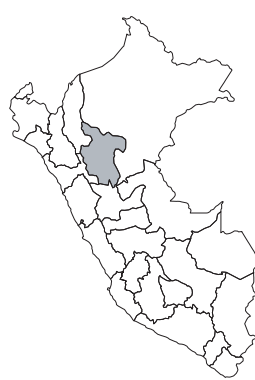

\section{EN, B1a}

Publicación: J. Wash. Acad. Sci. 22: 127. 1932.

Colector: L. Williams 7701 (F, US)

Nombre común: Desconocido.

Registro departamental: SM.

Regiones Ecológicas: BMHM, BMHP; $1350-1500 \mathrm{~m}$.

SINANPE: Sin registro.

Herbarios peruanos: Ninguno.

Observaciones: Arbusto conocido del área entre las cuencas del Sisa y del Mayo. Aparentemente no ha vuelto a ser recolectada desde 1930

\section{Streblacanthus amoenus (Bremek.) T.F. Daniel}

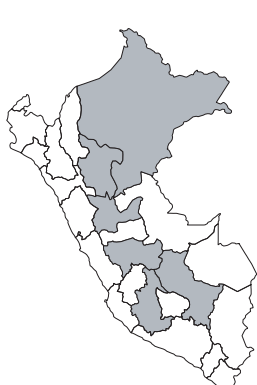

\section{LC}

Publicación: Novon 6(2): 147. 1996. Colector: R.C.B. van den Brink 1402 (U) Nombre común: Desconocido. Registro departamental: AY, CU, HU, JU, LO, SM.

Regiones Ecológicas: BHA; $250-800 \mathrm{~m}$. SINANPE: PNTM

Herbarios peruanos: Ninguno.

Observaciones: Este arbusto fue descrito de una planta cultivada en Indonesia de la que hasta 1996 se desconocía su procedencia. Daniel (1996) aclaró el origen y distribución de esta especie peruana, la cual ha sido recolectada en varias localidades del oriente del país. Esta especie se comercializa mundialmente por su valor ornamental.

36. Suessenguthia vargasii Wassh. var. hirsuta SchmidtLebuhn

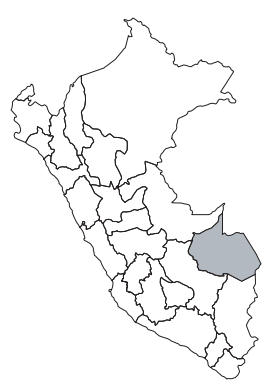

$$
\text { CR, B1a }
$$

Publicación: Candollea 58(1): 116-118, f. 9. 2003.

Colector: A.H. Gentry et al. 51566 (MO, NY; AMAZ!)

Nombre común: Desconocido.

Registro departamental: MD.

Regiones Ecológicas: BHA; 270—280 m.

SINANPE: PNBS, RNT

Herbarios peruanos: AMAZ (isotipo), USM (1).
Observaciones: Arbusto trepador conocido de una localidad en el sur-oriente del país. Esta especie fue recolectada en la Reserva Nacional Tambopata y es posible que otras subpoblaciones se encuentren en el Parque Nacional Bahuaja-Sonene. Esta zona requiere de estudios florísticos detallados.

\section{Tetramerium denudatum T.F. Daniel}

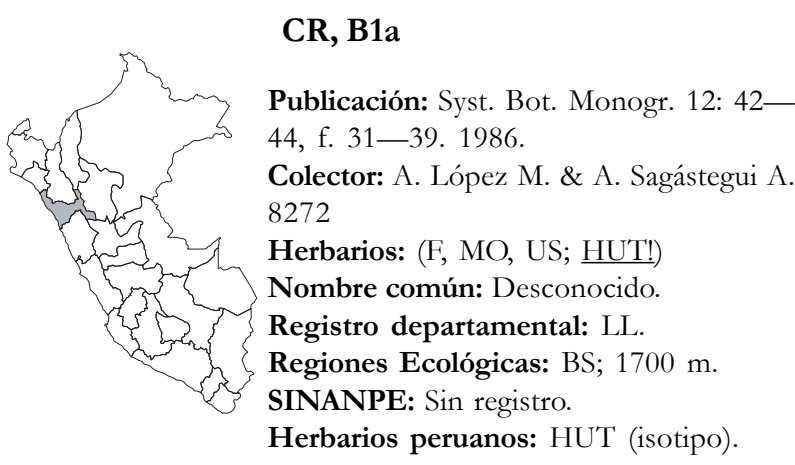

Observaciones: Esta especie herbácea crece postrada en laderas pedregosas de la cuenca del Marañón. Aparentemente no ha vuelto a ser recolectada desde 1974. Esta parte de la cuenca requiere de un estudio florístico detallado, pues alberga varios endemismos. Esta zona está fuertemente influenciada por la actividad minera y actividades relacionadas.

\section{Tetramerium peruvianum (Lindau) T.F. Daniel}

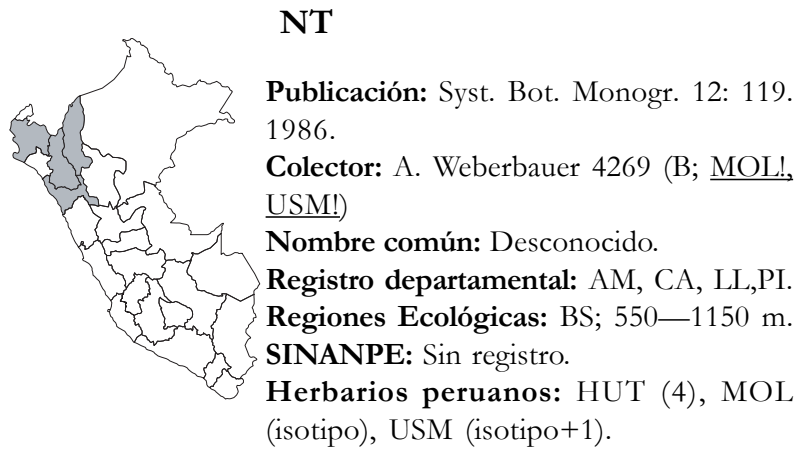

Observaciones: Esta especie herbácea o subarbustiva es conocida de cinco localidades en el norte del país en las cuencas del Chotano, Chamaya, Huancabamba y Marañón. Sus poblaciones ocupan ambientes rocosos y tal vez este ambiente agreste las proteja del pastoreo intensivo.

\section{Tetramerium sagasteguianum T.F. Daniel}

\section{EN, B1ab(iii)}

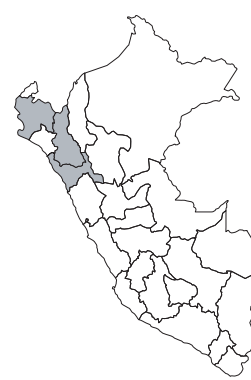

Publicación: Syst. Bot. Monogr. 12: 110113, f. 6, 8, 39, 125-129. 1986.

Colector: T.F. Daniel \& M.L. Butterwick 4319 (ASU, BM, F, GH, K, MICH, MO, NY, UC, US; HUT!)

Nombre común: Desconocido.

Registro departamental: CA, LL, PI.

Regiones Ecológicas: MDE, BS; 1000$1815 \mathrm{~m}$.

SINANPE: Sin registro.

Herbarios peruanos: HAO (3), HUT (isotipo+3).

Observaciones: Esta especie se conoce solamente de la vertiente del Pacífico en el norte del país. Al menos en la localidad del tipo, ubicada en la cuenca del Magdalena, parece ser frecuente. Amenazas a las poblaciones de esta especie provienen de los incendios intencionales. 


\section{Tetramerium surcubambense T.F. Daniel}

\section{CR, B1ab(iii)}

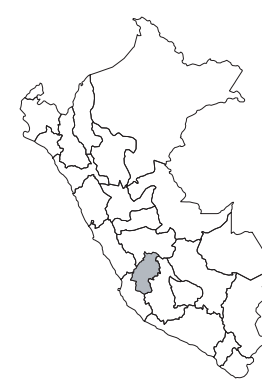

Publicación: Syst. Bot. Monogr. $12: 44$ 46, f. 39-42. 1986

Colector: A. Weberbauer 6483 (F, GH, US; MOL!, USM!)

Nombre común: Desconocido.

Registro departamental: HV.

Regiones Ecológicas: BS; $1500 \mathrm{~m}$.

SINANPE: Sin registro.

Herbarios peruanos: MOL (isotipo), USM (isotipo).

Observaciones: Esta planta leñosa es conocida de la colección tipo, una planta recolectada en la cuenca del Mantaro en 1913. Esta parte de la cuenca alberga ambientes ecotonales de bosque montano húmedo con matorrales semixéricos (Weberbauer, 1945). La localidad original ha sido escasamente herborizada aun cuando alberga varios endemismos. Para la conservación de esta especie deberá considerarse el impacto de pastoreo caprino y probablemente incendios intencionales.

\section{Tetramerium zeta T.F. Daniel}

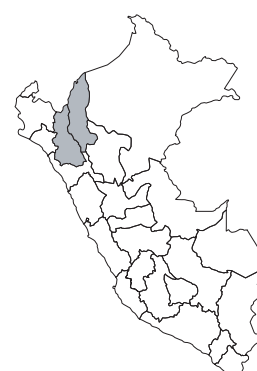

\section{EN, B1ab(iii)}

Publicación: Syst. Bot. Monogr. 12: 6669, f. 39, 60-62. 1986.

Colector: D. Wasshausen \& F. Encarnación 927

Herbarios: (US)

Nombre común: Desconocido

Registro departamental: AM, CA.

Regiones Ecológicas: BS; 350—700 m.

SINANPE: Sin registro.

Herbarios peruanos: USM (1).

Observaciones: Esta hierba perenne es conocida aparentemente de dos localidades, ambas en la cuenca del Marañón, recolectadas en la década de 1970. Esta zona requiere de estudios botánicos detallados, pues alberga varios endemismos. Probablemente pastoreo caprino y expansión rural podrían ser problemas para los hábitats mésicos en que crece esta especie.

\section{Trichosanchezia chrysothrix Mildbr.}

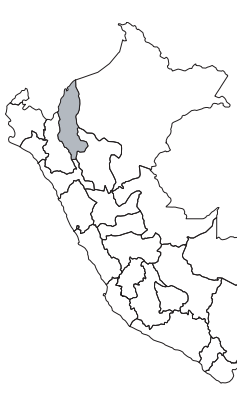

EN, B1a

Publicación: Notizbl. Bot. Gart. BerlinDahlem 9: 984-985. 1926.

Colector: G. Tessmann 4011 (B)

Nombre común: Desconocido.

Registro departamental: AM.

Regiones Ecológicas: BHA; 370-1000

$\mathrm{m}$.

SINANPE: Sin registro.

Herbarios peruanos: HUT (1).

Observaciones: Hierba o subarbusto conocido del nor-oriente del país, de las cuencas del Santiago y del Imaza. Esta especie fue descrita de una planta recolectada en 1924 en la cuenca del Santiago, en la cual no ha vuelto a ser hallada. Poco se sabe de sus poblaciones, aunque algunas de estas han sido registradas hasta 1997. 